\title{
Diagnostic algorithm utilising multimodal imaging including optical coherence tomography angiography for the detection of myopic choroidal neovascularisation
}

\author{
Akanksha Bagchi $^{1} \cdot$ Roy Schwartz $\mathbb{1}^{1} \cdot$ Philip Hykin $^{1} \cdot$ Sobha Sivaprasad (1) ${ }^{1}$ \\ Received: 14 October 2018 / Revised: 4 February 2019 / Accepted: 10 February 2019 / Published online: 26 February 2019 \\ (c) The Royal College of Ophthalmologists 2019
}

\begin{abstract}
Purpose To develop a diagnostic algorithm in patients with pathologic myopia who present with typical symptoms or signs of myopic choroidal neovascularisation (mCNV).

Methods Retrospective study. Patients with high myopia and suspected mCNV underwent fluorescein angiography (FFA), structural spectral-domain optical coherence tomography (SD-OCT) and optical coherence tomography angiography (OCTA). Active $\mathrm{mCNV}$ on one imaging modality plus clinical features were considered as the benchmark reference for the other two tests. Sensitivity was calculated for each modality individually and in combination. Morphological features were noted on SD-OCT and OCTA.

Results Twenty-seven eyes of 26 patients were analysed. Sensitivity of SD-OCT or FFA alone was 85.19\% (23/27 eyes). Sensitivity of OCTA was $74.07 \%$ (20/27 eyes). The sensitivity for SD-OCT combined with OCTA was $96.16 \%$ and combined with FFA was $97.80 \%$. On OCTA, a "tight net" appearance was seen in 16 eyes (80\%); a core vessel was visible in seven eyes (35\%), all with active lesions. A "perilesional halo" was visible in 11 eyes (55\%) of which 10 had active lesions.

Conclusion When combined, OCTA and SD-OCT or SD-OCT and FFA showed similar higher sensitivities than each modality alone. A tight vascular net and the combination of a perilesional halo and a visible core on OCTA may serve as biomarkers of mCNV activity.
\end{abstract}

\section{Introduction}

Pathologic myopia is defined as a refractive error of more than -6 dioptres or an axial length greater than $26 \mathrm{~mm}$ [1]. The prevalence of pathologic myopia ranges between 0.9 and $3.1 \%$ of the general population [2,3]. The most significant complication of pathologic myopia is myopic choroidal neovascularisation (mCNV), seen in $4-11 \%$ of patients with this condition [4, 5]. Fluorescein Angiography (FFA) has the advantage of detecting blood flow in real time and is the gold standard imaging modality for diagnosing

These authors contributed equally: Akanksha Bagchi, Roy Schwartz.

Akanksha Bagchi

akanksha.bagchi@gmail.com

1 NIHR Biomedical Research Centre, Moorfields Eye Hospital, London, UK
$\mathrm{mCNV}$ [6]. It is able to detect small lesions, allowing for early initiation of treatment [7]. However, it is a lengthy and invasive procedure with a small, albeit potentially lifethreatening, risk of anaphylaxis among other possible side effects [8].

Multimodal imaging has been used in recent times to aid in the diagnosis of mCNV. Recent studies found higher agreement in the diagnosis of $\mathrm{mCNV}$ when FFA was combined with spectral-domain optical coherence tomography (SD-OCT) [1, 9]. However, structural SD-OCT Bscan does not detect blood flow and is not useful in differentiating active from inactive $\mathrm{mCNV}$ in the absence of macular fluid [10].

Optical coherence tomography angiography (OCTA) is a novel and non-invasive technique for demonstrating the microvascular blood flow within the retina. It produces depth-resolved evaluation of the reflectance data from retinal tissue, providing a three-dimensional volume of information [11]. Unlike FFA, OCTA cannot detect dye leakage, a marker of vascular permeability. However, it is able to 
identify an increase in vascularity in a a three-dimensional manner.

As each of these modalities have their limitations, development of an algorithm to diagnose mCNV may aid in the diagnosis of this condition.

The purpose of this study was to develop a diagnostic algorithm by comparing the sensitivity of FFA, structural SD-OCT and OCTA as single diagnostic modalities or in combination in patients with pathologic myopia who present with typical symptoms.

\section{Materials and methods}

This retrospective study was approved by the Moorfields Review Board (ROAD 17/024). The study followed the tenets of the Declaration of Helsinki.

Eligibility criteria included patients with high myopia (defined as $\mathrm{AL}>26 \mathrm{~mm}$ or a refractive error $>-6$ diopters) who presented to the medical retina clinic at Moorfields Eye Hospital with new onset of visual disturbance, blurring of vision, or the appearance of scotoma, or clinical signs raising the suspicion of $\mathrm{mCNV}$, including the appearance of a grey elevated lesion or a haemorrhage at the posterior pole seen on biomicroscopy. We included all eligible patients between November 2016 and July 2017 and for whom all three tests were done. Eyes with clinical signs or symptoms of $\mathrm{mCNV}$ plus active mCNV on any one imaging modatity were considered as the benchmark referrence for the other two tests.

Exclusion criteria included poor quality of images, lack of one of the imaging modalities, dome shaped or traction maculopathy-diagnosis was made by senior ophthalmologist (S.S) and presence of any co-existing ocular condition other than myopia, including ocular inflammation, vitreoretinal disorders and glaucoma.

All patients underwent a thorough ophthalmologic evaluation including visual acuity testing, a detailed slit lamp examination after mydriasis, FFA, structural SD-OCT Bscan and OCTA.

FFA was performed on HRA-2 (Heidelberg Engineering, Heidelberg, Germany) by experienced technicians as per routine clinical practice. Images were analysed for presence of $\mathrm{mCNV}$ defined by the presence of early hyperfluorescence that increased in late frames or an area of late leakage. Blocked fluorescence due to blood or pigmentation was also recorded.

Structural SD-OCT B-scan was performed on the Spectralis system (Spectralis; Heidelberg Engineering, Heidelberg, Germany) with enhanced depth imaging technique wherever possible. The protocol used in clinical practice was a $3 \mathrm{D}$ volume set of $10 \times 10^{\circ}(2.9 \times 2.9 \mathrm{~mm})$ of the macula, consisting of 512 section images, and an Automatic Real-Time mode (ART) of seven frames per scan. Scans were included if quality allowed for interpretation of findings. Myopic CNV was defined as an area of homogenous hyper-reflectivity either below or above the RPE, and categorized as Type 1 or 2 , respectively. Other features noted on structural SD-OCT B-scan were intactness of the ellipsoid zone, presence or absence of sub- or intra-retinal fluid and visibility of the external limiting membrane (ELM). The CNV was considered active in the presence of sub-retinal hyper-reflective material (SHRM) and/or subretinal and/or intra-retinal fluid.

OCTA images were captured on the AngioPlex CIRRUS HD-OCT Model 5000 (Carl Zeiss Meditec, Inc., Dublin, USA) centred on the fovea. We analysed the $3 \times 3 \mathrm{~mm}$ angio cube protocol, which contains 245 B-scan slices repeated up to four times at each B-scan position. Each Bscan is made up of 245 A-scans, each A-scan is 1024 pixels deep. The automated protocol of the AngioPlex displays the vascular structure of choroid, choriocapillaris, deep retinal layers and superficial retinal layers. The quality of the images was deemed satisfactory for analysis if the image quality score was $>6$. The automated segmentation was adjusted manually to achieve the best plane to identify the mCNV. After correction of contrast and brightness, the presence of $\mathrm{mCNV}$ was identified as a bright interlacing or tangled group of vessels.

The following characteristics were included in the analysis: The location of the mCNV was identified as foveal or non-foveal involving $\mathrm{mCNV}$, a description of the mCNV as either a "tight net"-described as a group of bright close knit branching vessels with sharp borders and/or vascular inter-connections, or a "loose net"-with an indistinct branching or filamentous pattern and/or presence of a thick mature looking vessel and a lack of a clear border. Presence of a core feeder vessel and the absence or presence of a perilesional hypo-reflective halo (previously described as regions of choriocapillaris alteration, corresponding to flow impairment, steal or localized atrophy surrounding the mCNV) were also noted [12, 13]. (Fig. 1).

Analysis of all images and scans was performed independently by two graders (A.B and R.S). All discrepancies were then adjudicated by a third senior grader (S.S).

\section{Statistical analysis}

All statistical analyses were performed using MedCalc software version 15.2.2 (MedCalc Software bvba, Belgium). Descriptive statistics of the patient characteristics were performed. Sensitivity with $95 \%$ confidence intervals was calculated, using the signs and symptoms as a reference standard. The true positives (TP), false positives (FP) and false negatives (FN) were calculated. Sensitivity indicated the ability of imaging not to miss activation of the disease $($ sensitivity $=\mathrm{TP} / \mathrm{TP}+\mathrm{FN})$. Serial sensitivity studies were 
Fig. 1 Multimodal imaging of myopic choroidal

neovascularisation $(\mathrm{mCNV})$ in a 27-year-old woman. a Early frame on fluorescein angiography (FFA) (47 s), showing a central hyperfluorescent lesion. b late frame of the same FA (4 min) showing leakage from the mCNV. c Optical coherence tomography showing a Type 2 choroidal neovascular lesion with sub-retinal fluid and distorted external limiting membrane (yellow arrow); $\mathbf{d}$ Optical coherence tomography angiography showing a loose net with loops and surrounding halo (asterix) (color figure online)
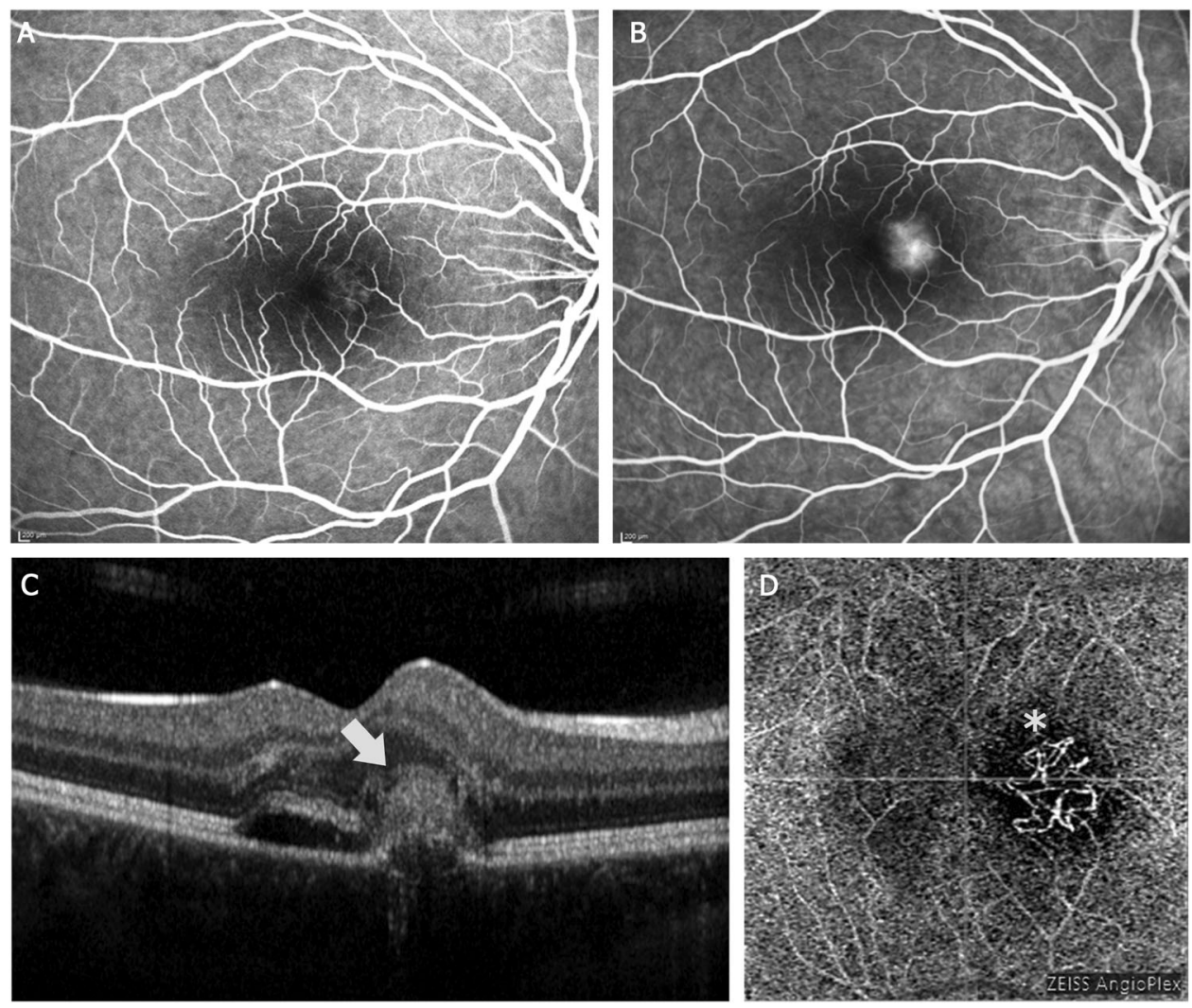

performed for SD-OCT and FFA and SD-OCT and OCTA in combination. Serial studies are defined as studies performed sequentially, with the second study dependent on the results of the first.

Clusters of findings and tests (as above) were also tabulated to determine the combinations that improved diagnostic accuracy. All possible combinations were examined to capture those factors that determined the strongest diagnostic accuracy.

\section{Results}

Twenty-seven eyes of 26 patients met the eligibility criteria and were included in the study. The mean age was $47.7 \pm$ 19.7 years. The majority of patients were female (18 (66\%)). Twenty-one (77\%) patients were Caucasian, 3 (11\%) Indian/Asian, 1 (3\%) Japanese, 1 (3\%) Chinese and 1 (3\%) Afro-Caribbean. Three patients had previously received bevacizumab injections to the fellow eye for mCNV.

\section{Imaging findings}

Table 1 lists findings for each patient on each imaging modality. Twenty patients had a positive OCTA, 23 had a positive SD-OCT and 23 had a positive FFA.
The morphological features of the neovascular complex noted on SD-OCT were as follows: The $\mathrm{mCNV}$ was type 1 in two eyes (7.4\%) and type 2 in 25 eyes (92.6\%). Subretinal fluid was present in 16 eyes $(69.6 \%)$, intra-retinal fluid in seven eyes $(30.4 \%)$, sub-retinal hyper-reflective material in 14 eyes $(60.9 \%)$, the ELM was absent in a total of 25 eyes $(92.6 \%)$ and in all except four eyes, its location corresponded to the area of leakage on FA. The ellipsoid layer was disrupted in 25 eyes $(92.6 \%)$.

The following features on OCTA were noted: The mCNV had the appearance of a "tight net" in 16 eyes (80\%) and a "loose net" in four eyes (20\%). A core vessel was visible in seven eyes (35\%). A hypo-reflective "perilesional halo" was visible in 11 eyes (55\%) of which 10 eyes corresponded with leakage on FFA and active $\mathrm{CNV}$ on SDOCT. The CNV was foveal in eight eyes (40\%) and extrafoveal in 12 eyes $(60 \%)$.

On FFA, there was early hyperfluorescence and late leakage seen in 23 eyes (85.19\%), staining was seen in two eyes $(7.41 \%)$ and no leakage was seen in two eyes $(7.41 \%)$.

\section{Sensitivity and specificity calculation}

Using clinical signs or symptoms and findings suggestive of active $\mathrm{mCNV}$ on at least one imaging modality as a benchmark, sensitivity of detection of mCNV on FFA, OCTA and structural SD-OCT B-scan was calculated. 
Table 1 Findings on different imaging modalities for 27 eyes of symptomatic patients with suspected myopic choroidal neovascularisation

\begin{tabular}{|c|c|c|c|c|c|c|}
\hline \multirow[t]{2}{*}{ No. } & \multirow[b]{2}{*}{ CNV Seen } & \multicolumn{2}{|l|}{ OCTA } & \multicolumn{2}{|l|}{ SD-OCT } & \multirow[t]{2}{*}{ FFA } \\
\hline & & Location & Morphology & Signs of activity ${ }^{a}$ & CNV type & \\
\hline 1 & Yes & Extra-foveal & Tight net & Active & Type 2 & Late leakage \\
\hline 2 & No & NA & NA & Active & Type 2 & No leakage \\
\hline 3 & No & NA & NA & Active & Type 2 & Late leakage \\
\hline 4 & Yes & Extra-foveal & Tight net & Active & Type 2 & Late leakage \\
\hline 5 & Yes & Foveal & Tight net & Inactive & Type 2 & Late leakage \\
\hline 6 & No & NA & NA & Active & Type 2 & Staining \\
\hline 7 & Yes & Extra-foveal & Tight net & Active & Type 2 & Late leakage \\
\hline 8 & Yes & Extra-foveal & Tight net & Active & Type 1 & Late leakage \\
\hline 9 & No & NA & NA & Active & Type 2 & Late leakage \\
\hline 10 & Yes & Extra-foveal & Loose net & Active & Type 1 & Late leakage \\
\hline 11 & No & NA & NA & Inactive & Type 2 & Late leakage \\
\hline 12 & Yes & Foveal & Tight net & Active & Type 2 & Late leakage \\
\hline 13 & Yes & Foveal & Tight net & Inactive & Type 2 & Late leakage \\
\hline 14 & Yes & Extra-foveal & Tight net & Inactive & Type 2 & Late leakage \\
\hline 15 & Yes & Extra-foveal & Tight net & Active & Type 2 & Late leakage \\
\hline 16 & Yes & Foveal & Loose net & Active & Type 2 & Late leakage \\
\hline 17 & Yes & Foveal & Loose net & Active & Type 2 & Staining \\
\hline 18 & Yes & Extra-foveal & Loose net & Active & Type 2 & Late leakage \\
\hline 19 & Yes & Extra-foveal & Tight net & Active & Type 2 & Late leakage \\
\hline 20 & Yes & Foveal & Tight net & Active & Type 2 & Late leakage \\
\hline 21 & No & NA & NA & Active & Type 2 & No leakage \\
\hline 22 & No & NA & NA & Active & Type 2 & Late leakage \\
\hline 23 & Yes & Extra-foveal & Tight net & Active & Type 2 & Late leakage \\
\hline 24 & Yes & Extra-foveal & Tight net & Active & Type 2 & Late leakage \\
\hline 25 & Yes & Extra-foveal & Tight net & Active & Type 2 & Late leakage \\
\hline 26 & Yes & Foveal & Tight net & Active & Type 2 & Late leakage \\
\hline 27 & Yes & Extra-foveal & Tight net & Active & Type 2 & Late leakage \\
\hline
\end{tabular}

OCTA optical coherence tomography angiography, $S D-O C T$ spectral-domain optical coherence tomography, $F F A$ fundus fluorescein angiography, $m C N V$ myopic choroidal neovascularisation

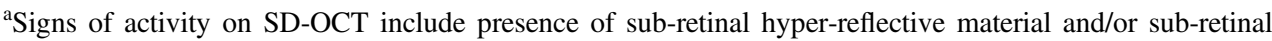
and/or intra-retinal fluid
Specificity calculation was not calculated as all eyes had an active mCNV lesions confirmed on at least one imaging modality. When considering one diagnostic modality only, twenty-three of 27 eyes demonstrated CNV on FFA alone. Sensitivity of FFA was $85.19 \%$ (CI 66.27-95.81\%). SDOCT B-scan identified an active CNV in 23 of 27 eyes. Sensitivity of SD-OCT was $85.19 \%$ (CI 66.27-95.81\%). An active mCNV was detected on OCTA in 20 eyes. Sensitivity of OCTA was $74.07 \%$ (CI $53.72-88.89 \%$ ).

As structural SD-OCT is usually the first diagnostic modality that is done in clinic, the combined sensitivity for the combination of SD-OCT with OCTA and SD-OCT with FFA was calculated compared to SD-OCT. The sensitivity of detecting $\mathrm{CNV}$ on SD-OCT combined with OCTA was $96.16 \%$ and that of SD-OCT combined with FFA was $97.80 \%$.

\section{Discussion}

In this study, we aimed to compare the sensitivity of structural SD-OCT B-scan, OCTA and FFA as single tools or in combination for the detection of $\mathrm{mCNV}$ and provide the unique characteristics of each of these modalities. We found that, when compared with clinical signs and symptoms, structural SD-OCT B-scan and FFA both have a high sensitivity $(85.19 \%)$ for the detection of $\mathrm{mCNV}$ in comparison with OCTA alone (74.07\%). Serial testing with SDOCT followed by OCTA or FFA resulted in increased sensitivities.

FFA is an important tool in the detection of mCNV [4]. Indeed, previous studies have demonstrated a greater detection rate for FFA in comparison with structural SDOCT B-scan for diagnosis of this entity $[1,6]$. However, it 
has several limitations including patient discomfort, the length of the exam, and possible side effects, including anaphylactic reactions. Moreover, the angiographic characteristics alone may lead to a misinterpretation of diagnosis as compared with the combination of FFA with more modern modalities such as SD-OCT [9].

Indeed, in the current study, FFA failed to detect the presence of a $\mathrm{mCNV}$ in four cases. In two of them, the false negative result stemmed from the presence of masking caused by submacular haemorrhage. This limitation is especially important in myopic patients, where haemorrhage may present a differential diagnosis between a mCNV and lacquer cracks, and imaging serves as a crucial tool in this distinction.

OCTA has several advantages over FFA and structural SD-OCT B-scan. Unlike the two-dimensional FFA, it provides a three-dimensional view of the retinal microcirculation. It also demonstrates in finer detail the morphological characteristics of choroidal neovascularization, which is usually masked by fluorescein leakage. Additionally, unlike structural B-scan SD-OCT it can detect the vascularity of the lesion, rather than just demonstrate a hyper-reflective material.

Previous studies investigated the sensitivity and specificity of OCTA in the detection of mCNV (Table 2). In a study by Querques et al., the sensitivity was $90.48 \%$ and specificity $93.75 \%$ [13]. In a paper by Bruyere et al., OCTA alone showed a $90 \%$ sensitivity for mCNV detection [14]. Specificity values were not reported. In a study by Miyata et al., sensitivity was $94.1 \%$, and specificity $100 \%$ [7].

The current study demonstrated a lower sensitivity than previously reported. Several reasons may account for these differences. On further investigation of undetected cases, we found that the area of mCNV measured on FFA was extremely small (the smallest lesion was $<0.07 \mathrm{~mm}^{2}$ ). It is possible that this small size does not allow for proper signal detection of the mCNV by OCTA. The study by Bruyere et al. stressed the ability of OCTA to detect small-sized lesions [14]. However, the mean mCNV area in their study was $0.22 \mathrm{~mm}^{2}$. Differences may also result from the different devices used in this versus the previous study (Zeiss Angioplex vs. AngioVue RTVue XR Avanti, respectively).

Another possible explanation is a slow flow within the $\mathrm{mCNV}$. Since OCTA relies on change between two consecutive B-scans, it only detects flow higher than a minimal threshold, the slowest detectable flow, determined by the time between the two B-scans [10]. Third, the presence of haemorrhage prevented OCTA from detecting the $\mathrm{mCNV}$ in the two eyes with submacular haemorrhage. This has been described previously as a barrier to CNV detection by OCTA [10]. Finally, retinal alterations resulting from myopic maculopathy, including retinal pigment epithelium (RPE) and chorioretinal atrophy, have been shown to interfere with the detection of mCNV using OCTA [13].

In the current study, structural SD-OCT had a sensitivity of $85.19 \%$ for the detection of $\mathrm{mCNV}$, suggesting that similar to OCTA, its use a single tool for the detection of $\mathrm{mCNV}$ is not sufficient. A previous study by Leveziel et al. reported that the exudative features of $\mathrm{mCNV}$ are more obvious on FFA than on structural SD-OCT B-scan [6]. However, its utility as a necessary adjunct to FFA has also been demonstrated. A study by Chhablani et al. conducted on 80 myopic eyes of 57 patients showed that, in comparison with FFA, structural SD-OCT had a better specificity, as well as negative and positive predictive values for the detection of $\mathrm{mCNV}$, resulting from possible confusion when interpreting FFA due to associated staining and posterior pole changes [1].

Despite the inherent limitations of each single modality, serial examination with other modalities increased the sensitivity significantly. Furthermore, using completely noninvasive imaging modalities (SD-OCT followed by OCTA) proved to be almost as sensitive as adding an invasive diagnostic technique (SD-OCT followed by FFA), (96.16\% vs $97.8 \%$, respectively).

Based on these data we propose an algorithm for the diagnosis of $\mathrm{mCNV}$ in real world practice settings (Fig. 2). SD-OCT as a first diagnostic modality will establish diagnosis in $85.19 \%$ of mCNV in symptomatic patients. If available, OCTA can improve the true-positive rates and confirm suspicion of mCNV. If, however, SD-OCT and OCTA are both negative or the diagnosis is uncertain, only then is FFA warranted.

Despite the usefulness of OCTA as a non-invasive tool in the diagnosis of mCNV, it is worth mentioning a few situations where OCTA alone is not adequate and FFA should be used. First, as mentioned, small mCNVs may go undetected on OCTA. Further studies are needed to find the size cut-off beyond which OCTA detection improves. Second, mCNVs involving a submacular haemorrhage may lead to a false-negative detection on OCTA due to masking. Unfortunately, the same masking can occur with FFA. Third, in an eye with advanced myopic pathologic changes (i.e. large areas of atrophy) OCTA may not be the best tool for $\mathrm{mCNV}$ detection.

The most prominent morphological pattern in the current study was a "tight" network pattern seen in $80 \%$ of cases. The "loose" network configuration was associated with a previous history of distortion in two patients. Similarly, in the study by Bruyere et al., there was a correlation between "tighter" networks (designated an "interlacing" pattern in that study) and treatment-naïve eyes. This is further demonstrated by the study by Querques et al. [13], where the interlacing pattern was more closely associated with mCNV activity and was the presenting pattern in all 
Table 2 Comparison of sensitivity and specificity values for the detection of myopic choroidal neovascularisation using optical coherence tomography angiography in different studies

Fig. 2 Decision making flow chart for clinical suspicion of myopic choroidal neovascularisation. $F A$ fundus fluorescein angiography, $S D$ $O C T$ optical coherence tomography, OCTA optical coherence tomography angiography, $m C N V$ myopic choroidal neovascularisation

\begin{tabular}{lllll}
\hline & Miyata et al. [7] & Bruyere et al. [14] & Querques et al. [13] & Current study \\
\hline Sensitivity of OCTA & $94.1 \%$ & $90 \%$ & $90.48 \%$ & $74.07 \%$ \\
Specificity of OCTA & $100 \%$ & NA & $93.75 \%$ & NA \\
\hline
\end{tabular}

OCTA optical coherence tomography angiography, NA Not applicable

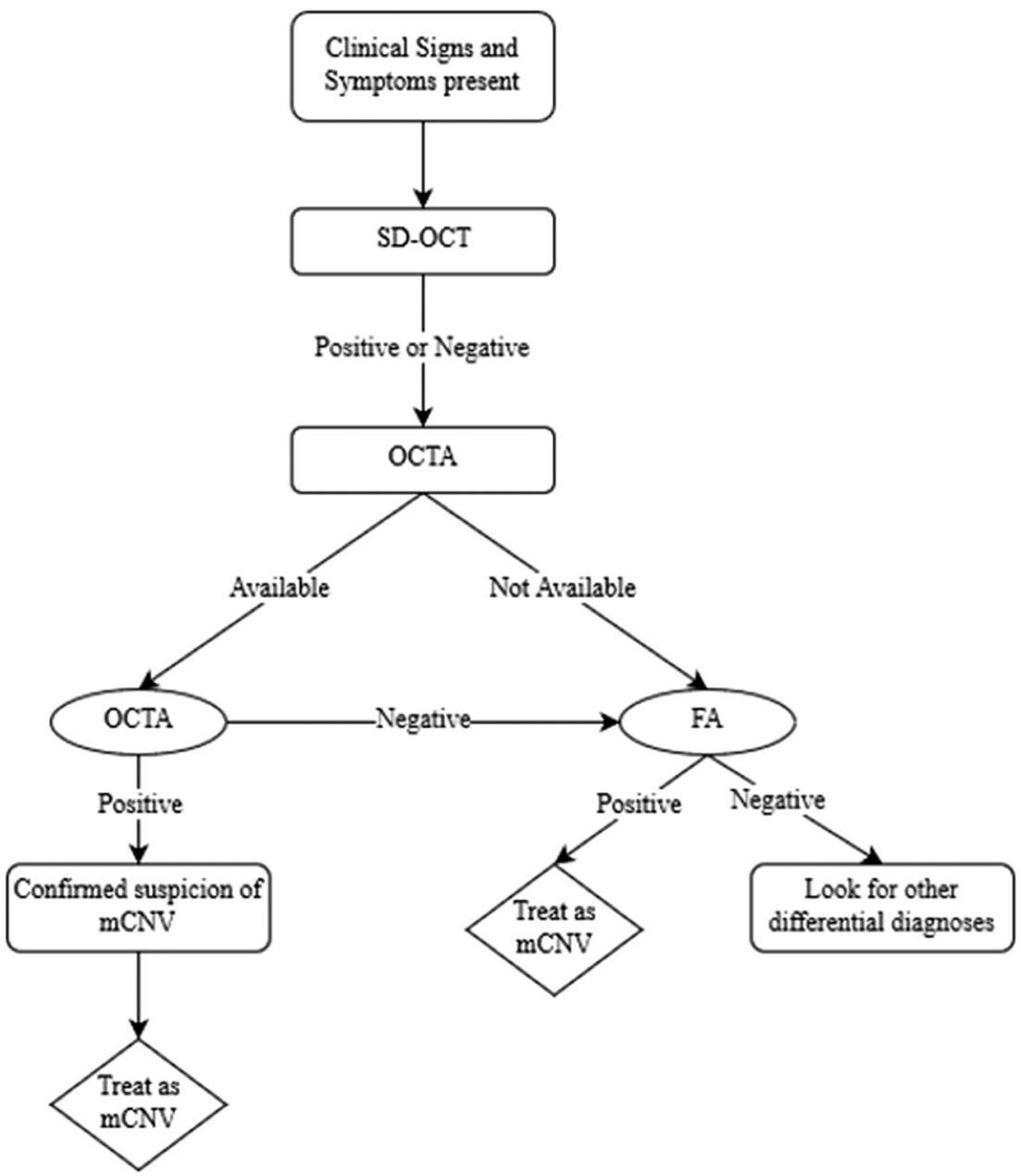

treatment-naïve patients. As all patients in the current cohort were treatment naïve, it is possible that a tighter vascular network is correlated with a newly-discovered mCNV. The fact that half of the patients in the current cohort with a loose net morphology had previous symptoms of distortion supports this theory.

A perilesional hypo-reflective halo was seen in 11 eyes on OCTA and 10 of them (90.91\%) had confirmed active mCNV on both SD-OCT and FFA. Similarly, a core vessel was visible in seven $(35 \%)$ eyes and interestingly, all seven eyes $(100 \%)$ showed active $\mathrm{mCNV}$ on SD-OCT and six $(85.71 \%)$ eyes corresponded with leakage on FFA. These observations may prove as helpful biomarkers in the diagnosis and monitoring of active mCNV lesions. In the current study, the ELM was absent in 25 eyes (92.6\%) and in
21 of them, its location corresponded to the area of leakage on FFA. In a previous study by Parodi et al. [15], a lack of ELM visibility was identified in all cases with leaking CNV on FFA and correlated with the site of FFA leakage. Both studies support this sign as an important biomarker of lesion activity. This may be a more important marker than intra- or sub-retinal fluid, as these do not always correlate with FFA leakage, as also demonstrated by the aforementioned study, wherein $10 \%$ of cases FFA leakage did not correspond to visible fluid accumulation on structural SD-OCT. However, it is worth mentioning Ding X et al. [16] reported the high sensitivity albeit low specificity of ELM interruption in the evaluation of $\mathrm{CNV}$ activity. Table 3 compares SD-OCT features with FFA leakage in both studies. Our study has several limitations. First, the cohort analyzed is relatively 
Table 3 Comparison of agreement between leakage (activity) on FFA with tomographic morphological features suggestive of active mCNV with a different study

\begin{tabular}{llll}
\hline \multicolumn{3}{l}{ OCT features } \\
\hline $\begin{array}{l}\text { Agreement of OCT findings } \\
\text { with leakage on FFA }\end{array}$ & SRF & IRF & $\begin{array}{l}\text { Absence of ELM } \\
\text { visibility }\end{array}$ \\
Parodi et al. [15] & $75 \%$ & $15.6 \%$ & $100 \%$ \\
Current study & $65.2 \%$ & $21.7 \%$ & $91.3 \%$ \\
\hline
\end{tabular}

FFA fundus fluorescein angiography, $O C T$ optical coherence tomography, $S R F$ sub-retinal fluid, IRF intra-retinal fluid, ELM external limiting membrane, $m C N V$ myopic choroidal neovascularisation

small. Second, the retrospective nature of this analysis. Finally, this study was not powered to detect the specificity of the different modalities in the detection of $\mathrm{mCNV}$, as all participants had a confirmed treatable lesion. However, we have assessed both single imaging multimodal imaging techniques and were able to suggest a diagnostic algorithm.

In conclusion, this study provides sensitivity values and activity biomarkers of different imaging modalities for the detection of mCNV. Although the sensitivity of OCTA, structural SD-OCT B-scan and FFA was high, none can be used safely as a single modality in the detection of mCNV. However, their combination aids in the diagnosis of this sometimes difficult to diagnose entity. Our findings support the presence of a tight vascular net, the combination of a perilesional halo and a visible core on OCTA as biomarkers of mCNV activity.

\section{Summary}

\section{What was known before}

- FFA is the gold standard test to diagnose myopic CNV. - Sensitivity of OCTA alone in diagnosing myopic CNV.

\section{What this study adds}

- Retrospective study analysing the sensitivity of FFA, SD-OCT and OCTA in diagnosing myopic CNV individually and in combination.

- An algorithm helping accurate diagnosis and guiding treatment is proposed.

- Morphological biomarkers of activity are suggested.

\section{Compliance with ethical standards}

Conflict of interest The authors declare that they have no conflict of interest.
Publisher's note: Springer Nature remains neutral with regard to jurisdictional claims in published maps and institutional affiliations.

\section{References}

1. Chhablani J, Deepa MJ, Tyagi M, Narayanan R, Kozak I. Fluorescein angiography and optical coherence tomography in myopic choroidal neovascularization. Eye. 2015;29:519-24. https://doi.org/10.1038/eye.2014.345.

2. Tufail A, Narendran N, Patel PJ, Sivaprasad S, Amoaku W, Browning AC. et al. Ranibizumab in myopic choroidal neovascularization: the 12-month results from the repair study. Ophthalmology. 2013;120:1944-6. https://doi.org/10.1016/j. ophtha.2013.06.010.

3. Wong TY, Ferreira A, Hughes R, Carter G, Mitchell P. Epidemiology and disease burden of pathologic myopia and myopic choroidal neovascularization: an evidence-based systematic review. Am J Ophthalmol. 2014;157:9-25. https://doi.org/10. 1016/j.ajo.2013.08.010. e12

4. Wong TY, Ohno-Matsui K, Leveziel N, Holz FG, Lai TY, Yu HG et al. Myopic choroidal neovascularisation: current concepts and update on clinical management. Br J Ophthalmol. 2015;99:28996. https://doi.org/10.1136/bjophthalmol-2014-305131.

5. Bandello F, Battaglia Parodi M. Antivascular endothelial growth factor for choroidal neovascularization in pathologic myopia. Dev Ophthalmol. 2010;45:73-83. https://doi.org/10.1159/000320010.

6. Leveziel Nicolas, Caillaux V, Bastuji-Garin S, Zmuda M, Souied E. Angiographic and optical coherence tomography characteristics of recent myopic choroidal neovascularization. Am J Ophthalmol. 2013;155:913. https://doi.org/10.1016/j.ajo.2012.11.021.e1.

7. Miyata M, Ooto S, Hata M, Yamashiro K, Tamura H, AkagiKurashige $\mathrm{Y}$ et al. Detection of myopic choroidal neovascularization using optical coherence tomography angiography. Am J Ophthalmol. 2016;165:108-14. https://doi.org/10.1016/j.ajo.2016. 03.009.

8. Kwiterovich KA, Maguire MG, Murphy RP, Schachat AP, Bressler NM, Bressler SB et al. Frequency of adverse systemic reactions after fluorescein angiography. Ophthalmology. 1991;98:1139-42. https://doi.org/10.1016/S0161-6420(91)321651.

9. Milani P, Massacesi A, Setaccioli M, Moschini S, Mantovani E, Ciaccia $S$ et al. Sensitivity of fluorescein angiography alone or with SD-OCT for the diagnosis of myopic choroidal neovascularization. Graefe's Arch Clin Exp Ophthalmol. 2013;251:18911900. https://doi.org/10.1007/s00417-013-2282-y.

10. De Carlo TE, Bonini Filho MA, Chin AT, Adhi M, Ferrara D, Baumal CR et al. Spectral-domain optical coherence tomography angiography of choroidal neovascularization. Ophthalmology. 2015;122:519-24. https://doi.org/10.1038/eye.2014.345.

11. Spaide RF, Fujimoto JG, Waheed NK, Science C. HHS Public Access. 2016. https://doi.org/10.1097/IAE.0000000000000765. Image.

12. Jia Y, Bailey ST, Wilson DJ, Tan O, Klein ML, Flaxel CJ et al. Quantitative optical coherence tomography angiography of choroidal neovascularization in age-related macular degeneration. Ophthalmology. 2015;121:1435-44. https://doi.org/10.1016/j. ophtha.2014.01.034.

13. Querques L, Giuffrè C, Corvi F, Zucchiatti I, Carnevali A, De Vitis LA et al. Optical coherence tomography angiography of myopic choroidal neovascularisation. Br J Ophthalmol. 2016: bjophthalmol-2016-309162. https://doi.org/10.1136/bjophtha lmol-2016-309162.

14. Bruyère E, Miere A, Cohen SY, Martiano D, Sikorav A, Popeanga A et al. Neovascularization secondary to high myopia imaged by 
optical coherence tomography angiography. Retina . 2017;37:2095101. https://doi.org/10.1097/IAE.0000000000001456.

15. Battaglia Parodi M, Iacono P, Bandello F. Correspondence of leakage on fluorescein angiography and optical coherence tomography parameters in diagnosis and monitoring of myopic choroidal neovascularization treated with bevacizumab. Retina. 2016;36:104-9. https://doi.org/10.1097/IAE.0000000000000684.
16. Ding X, Zhan Z, Sun L, Yang Y1, Li S1, Zhang A et al. Retinal pigmental epithelium elevation and external limiting membrane interruption in myopic choroidal neovascularization: correlation with activity. 2018. Graefes Arch Clin Exp Ophthalmol. 2018;256:1831-7. https://doi.org/10.1007/s00417018-4060-3. 